\title{
ST3Gal3 confers paclitaxel-mediated chemoresistance in ovarian cancer cells by attenuating caspase-8/3 signaling
}

\author{
XIAN ZHANG $^{1^{*}}$, XINYING YANG $^{2^{*}},{\text { MING } \text { CHEN }^{1}, \text { SHAOLIE ZHENG }}^{1}$, \\ JINYUAN LI ${ }^{3}$, SHAOQIANG LIN ${ }^{3,4}$ and XIAOYU WANG ${ }^{1}$
}

\author{
${ }^{1}$ Department of Obstetrics and Gynecology, The First Affiliated Hospital, Jinan University, Guangzhou, Guangdong 510630; \\ ${ }^{2}$ Guangdong Second People's Hospital, Guangzhou, Guangdong 510317; ${ }^{3}$ Institute of Clinical Medicine, \\ The First Affiliated Hospital, Jinan University, Guangzhou, Guangdong 510630; \\ ${ }^{4}$ Clinical Department of Scientific Research Center, The First Affiliated Hospital of Guangdong \\ Pharmaceutical University, Guangzhou, Guangdong 510080, P.R. China
}

Received December 12, 2018; Accepted August 8, 2019

DOI: $10.3892 / \mathrm{mmr} .2019 .10712$

\begin{abstract}
The aberrant expression of sialyltransferase has a role in cell differentiation, neoplastic transformation and the progression of various types of cancer. Our previous studies have shown that high expression of $\beta$-galactoside- $\alpha 2,3$-sialylt ransferase III (ST3Gal3) in the metastatic ovarian cancer cell line HO8910PM attenuated cisplatin-induced apoptosis. The present study demonstrated that paclitaxel-induced chemoresistance in ovarian cancer cells upregulated the expression of ST3Gal3 and reduced the activity of caspase- $8 / 3$. The results of the present study revealed that the endogenous levels of ST3Gal3 mRNA and protein were significantly higher in HO8910PM cells compared with SKOV3 cells. A higher expression of ST3Gal3 was correlated with an increased resistance to paclitaxel, while the downregulation of ST3Gal3 resulted in paclitaxel-induced apoptosis. Paclitaxel upregulated ST3Gal3 expression at the mRNA and protein levels in HO8910PM cells, but not in SKOV3 cells. Silencing of ST3Gal3 by small interfering RNA reversed these effects and increased the protein levels of caspase- $8 / 3$, which may contribute to paclitaxel-induced apoptosis. The results of the present study
\end{abstract}

Correspondence to: Professor Xiaoyu Wang, Department of Obstetrics and Gynecology, The First Affiliated Hospital, Jinan University, Building 4, 613 Huangpu West Road, Guangzhou, Guangdong 510630, P.R. China

E-mail: twxy163@163.com

Professor Shaoqiang Lin, Clinical Department of Scientific Research Center, The First Affiliated Hospital of Guangdong Pharmaceutical University, Building 1, 1 Gonghe West Street, Guangzhou, Guangdong 510080, P.R. China

E-mail: shaotsiang@163.com

*Contributed equally

Key words: $\beta$-galactoside- $\alpha 2,3$-sialyltransferase III, paclitaxel, ovarian cancer, chemoresistance suggested that ST3Gal3 was a target for paclitaxel-related resistance during ovarian cancer chemotherapy.

\section{Introduction}

Ovarian cancer remains one of the most aggressive and highly recurrent malignant diseases worldwide with a poor prognosis $(1,2)$. The vague clinical symptoms in the early stages of ovarian cancer have a major role in delaying intervention and treatment (3). Several diagnostic biomarkers have been identified for ovarian cancer. Serum human epididymis protein 4 (HE4) and transthyretin (TTR) were identified as novel biomarkers for ovarian cancer (4). Compared with the golden standard marker mucin-16 (CA125), HE4 is suitable for patients with advanced ovarian cancer, while TTR is a better serum biomarker for patients with early stage ovarian cancer (4). A panel of four serum biomarkers (CA125, HE4, E-cadherin and interleukin-6) has been shown to have a sensitivity of $95-100 \%$ for patients with early stage ovarian cancer (5). However, unlike the progression in the discovery of diagnostic biomarkers for ovarian cancer, biomarkers associated with more efficient therapeutic targets remain elusive.

Sialylation is one of the essential molecular posttranslational modifications, which has important roles in metabolism, immunity, development and cancer biology (6-9). In ovarian cancer, sialylation of glycoproteins is a common modification in ovarian cancer proximal fluids (10). Aberrantly sialylated N-linked glycopeptides may serve as serum biomarkers for patients with ovarian cancer (11-13). The fully sialylated $\alpha$-chain of complement 4-binding protein has been identified as a diagnostic and prognostic marker for ovarian cancer $(14,15)$. $\beta$-galactoside- $\alpha 2,3$-sialyltransferase I (ST3Gal1) was found to be expressed at a higher level in the advanced stage of epithelial ovarian cancer, and was demonstrated to facilitate epidermal growth factor receptor (EGFR) signaling and the migration and peritoneal dissemination of ovarian cancer cells (16). The $\alpha 2,6 \mathrm{~N}$-linked sialylation of the $\beta 1$ integrins was reported to promote cell adhesion and invasion of ovarian cancer cells (17). In addition, the volume of 
ascites indicated the occurrence of transmesothelial invasion, which correlated with a poorer prognosis for patients with ovarian cancer $(18,19)$. It has recently been reported that there is a positive correlation between the volume of ascites and the amount of serum sialylated structures in patients with epithelial ovarian cancer (8). Therefore, sialyltransferase-catalyzed sialylations are a prevalent and aggravating factor in ovarian cancer initiation and progression.

The treatment of ovarian cancer includes surgery, chemotherapy and immunotherapy (20). Due to the vague clinic symptoms in the early stages of ovarian cancer, most patients with ovarian cancer are identified when the malignancy becomes advanced, which reduces the availability of surgical intervention $(21,22)$. The first line chemotherapies used for ovarian cancer are predominantly platinum-based and taxane-based drugs $(22,23)$. Surgery in combination with chemotherapy or chemotherapy alone remain the standard care for advanced ovarian cancer, although most patients with advanced ovarian cancer develop chemoresistance, metastasis and bowel obstructions, which are the most frequent causes of mortality (22). The molecular mechanisms of chemoresistance are complex, including the maintenance of cancer stem cells, the aberrant activation of multi-drug resistant pathways, the aberrant activation of ABC transporters and oncogenic mutation (24-27). HE4 not only serves as a diagnostic marker for ovarian cancer, but is also a predictor of platinum sensitivity in ovarian cancer (28). $\alpha 2,6$ N-linked sialylated EGFR confers acquired resistance to gefitinib in ovarian cancer (29). ST6GAL1 confers cisplatin resistance in ovarian tumor cells (30). Our previous study showed that ST3Gal3 correlated with cisplatin resistance in ovarian tumor cells (31). In the present study, the relationship between ST3Gal3 expression and paclitaxel resistance in ovarian tumor cells was investigated in order to provide a better understanding of the effect of paclitaxel treatment alone or in combination with ST3Gal3.

\section{Materials and methods}

Cell culture. The human ovarian cell line SKOV3 was purchased from the American Type Culture Collection and the HO8910PM cell line was purchased from The Cell Bank of Type Culture Collection of The Chinese Academy of Sciences. SKOV3 cells were cultured in RPMI-1640 medium (Gibco; Thermo Fisher Scientific, Inc) containing 10\% FBS (Gibco; Thermo Fisher Scientific, Inc.) and $1 \%$ penicillin-streptomycin (Gibco; Thermo Fisher Scientific, Inc.). HO8910PM cells were cultured in DMEM high-glucose medium (Gibco; Thermo Fisher Scientific, Inc.) containing 10\% FBS and 1\% penicillin-streptomycin. All cell lines were cultured at $37^{\circ} \mathrm{C}$ in a humidified incubator with $5 \% \mathrm{CO}_{2}$.

RNA isolation and reverse transcription-quantitative $(R T-q) P C R$. Total RNA was extracted using TRIzol reagent (Invitrogen; Thermo Fisher Scientific, Inc.), according to the manufacturer's instructions. Total RNA was reverse transcribed using TransScript One-Step gDNA Removal and cDNA Synthesis SuperMix (Beijing Transgen Biotech Co., Ltd.). Complementary DNA was amplified using UltraSYBR Mixture (High ROX; CWBio) and the following primers: ST3Gal3 forward, 5'-AAAACGACACTGCGCATCAC-3' and reverse, 5'-TCGAGTGGCCACAGATTTCC-3'; and GAPDH forward, 5'-AGCCTCAAGATCATCAGC-3' and reverse 5'-GAGTCCTTCCACGATACC-3'. The qPCR cycling conditions were as follows: $95^{\circ} \mathrm{C}$ for $30 \mathrm{sec}$, followed by 40 cycles of $95^{\circ} \mathrm{C}$ for $5 \mathrm{sec}$ and $60^{\circ} \mathrm{C}$ for $40 \mathrm{sec}$. The relative levels of mRNA expression were normalized to GAPDH and calculated using the $2^{-\Delta \Delta \mathrm{Cq}}$ method (32).

Cell Counting Kit-8 (CCK-8) assays. SKOV3 and HO8910PM cells were seeded in 96 -well plates at a density of $5 \times 10^{3}$ cells/well. The next day, the cells were treated with $0,5,10,20,40,80$, 160 or $320 \mathrm{ng} / \mathrm{ml}$ paclitaxel (Sigma-Aldrich; Merck KGaA) or the equivalent volume of DMSO as a negative control. After $48 \mathrm{~h}$ of incubation, the cell viability was determined using the CCK-8 assay (Beyotime Institute of Biotechnology), according to the manufacturer's instructions. Cytotoxicity was calculated as follows: Cytotoxicity $(\%)=[1-($ optical density of tested cells)/(optical density of control cells)] x100. The $\mathrm{IC}_{50}$ (half maximal inhibitory concentration) value was calculated by GraphPad Prism 6 (GraphPad Software, Inc.).

Small interfering (si)RNA transfection and paclitaxel treatment. In total, three ST3Gal3 siRNA sequences were designed and synthesized by Guangzhou RiboBio Co., Ltd. The siRNA sequences for siCTRL was: 5'-TTCTCCGAA CGTGTCACGT-3'. The siRNA sequences for ST3Gal3 were: 1\#-sense 5'-CGTGGAAGCTACACTTACT-3', 2\#-sense 5'-CCTGAATCTGGACTCTAAA-3' and 3\#-sense 5'-CCT GGACGCACAATATCCA-3'. These siRNA sequences were tested in a previous study (31), and the 1\# siRNA sequence was then used for further experiments. Briefly, cells were seeded into 6 -well plates at a density of $2 \times 10^{5}$ cells/well. The next day, $7.5 \mu$ l Lipofectamine ${ }^{\circledR}$ RNAiMAX reagent (Invitrogen; Thermo Fisher Scientific, Inc.) was diluted in $125 \mu \mathrm{l}$ Opti-MEM (Invitrogen; Thermo Fisher Scientific, Inc.) and 30 pmol siRNA was diluted in $125 \mu \mathrm{l}$ Opti-MEM, and each was mixed by vortexing for $10 \mathrm{sec}$. The diluted siRNA was added to the diluted Lipofectamine ${ }^{\circledR}$ RNAiMAX Reagent and incubated for $10 \mathrm{~min}$ at room temperature. During the incubation, the cells were washed once with $3 \mathrm{ml}$ of PBS and $2 \mathrm{ml}$ fresh growth medium was added. Subsequently, the $250 \mu \mathrm{l}$ transfection mixture was added dropwise onto the cells in the 6-well plate, incubated for two days and then exposed to $20 \mathrm{ng} / \mathrm{ml}$ paclitaxel for a further $48 \mathrm{~h}$.

Western blot. Cells were collected and lysed on ice using RIPA lysis buffer (Beyotime Institute of Biotechnology) containing 1\% PMSF (Beyotime Institute of Biotechnology). The protein concentration was determined using the bicinchoninic acid method. In total, $50 \mu \mathrm{g}$ of total protein was separated using $10 \%$ SDS-PAGE and transferred onto PVDF membranes. After 5\% bovine serum albumin (cat. no. ST023; Beyotime Institute of Biotechnology) blocking at room temperature for $1 \mathrm{~h}$, membranes were incubated overnight at $4^{\circ} \mathrm{C}$ with primary antibodies. The primary antibodies used were: anti-ST3Gal3 (1:500 dilution; cat. no. SC-134040; Santa Cruz Biotechnology, Inc.), anti-caspase-8 (1:1,000 dilution; cat. no. 4790; Cell Signaling Technologies, Inc.), anti-caspase-3 (1:1,000 dilution; cat. no. 14220; Cell Signaling Technologies, Inc.) and anti-GAPDH (1:2,000 dilution; cat. no. AF0006; Beyotime 
Institute of Biotechnology). Membranes were then incubated with secondary anti-rabbit or anti-mouse horseradish peroxidase (HRP)-conjugated secondary antibodies (1:3,000 dilution; cat. nos. A0208 and A0216; Beyotime Institute of Biotechnology) for $1 \mathrm{~h}$ at room temperature. Results were acquired using the Gel Logic 1500 imaging system (Kodak). GAPDH was used as a loading control.

Flow cytometry for Maackia amurensis lectin II (MAL II) staining. Briefly, after the medium was discarded, cells were washed twice with PBS and digested with trypsin/0.25\% EDTA (Gibco; Thermo Fisher Scientific, Inc.). Fresh growth medium was added to terminate the digestion and cells were collected by centrifugation at $1,000 \mathrm{x}$ g for $5 \mathrm{~min}$ at room temperature. The cells were resuspended and washed twice in $1 \mathrm{ml}$ PBS. The cell pellets were resuspended in $100 \mu \mathrm{l}$ of HEPES containing $0.5 \% \mathrm{BSA}$ and $2.5 \mu \mathrm{g} / \mathrm{ml}$ biotinylated MALII (Vector Laboratories, Ltd.), and incubated at room temperature for $2 \mathrm{~h}$ in the dark. The cells were washed twice with PBS and incubated with $1 \mu \mathrm{g} / \mathrm{ml}$ streptavidin-phycoerythrin (Sigma-Aldrich; Merck KGaA) for $1 \mathrm{~h}$ at room temperature in the dark. After washing the stained cells twice with PBS, cell surface MAL II was quantified using a FACSCanto II flow cytometer (BD Biosciences), and analyzed by BD CellQuest Software version 3.3 (BD Biosciences) according to the manufacturer's instructions.

Flow cytometry for apoptosis analysis. Cells were digested and collected as aforementioned. Cells were resuspended and briefly washed twice in $1 \mathrm{ml}$ PBS. The final pellets were resuspended in $500 \mu \mathrm{l}$ of $1 \mathrm{X}$ binding buffer, and $5 \mu \mathrm{l}$ of Annexin-V-FITC (BD Biosciences) was added and incubated at room temperature for $15 \mathrm{~min}$ in the dark. Then $5 \mu \mathrm{l}$ of propidium iodide (BD Biosciences) was added to the cells and incubated at room temperature for $5 \mathrm{~min}$ in the dark. Apoptosis was immediately quantified using the FACSCanto II flow cytometer (BD Biosciences), and analyzed by BD CellQuest Software version 3.3 (BD Biosciences).

TUNEL assay. In brief, cells were fixed with $4 \%$ paraformaldehyde at room temperature for 20 min and washed twice with PBS. Cells were then permeabilized with $0.3 \%$ Triton $\mathrm{X}-100$ at room temperature for $10 \mathrm{~min}$, washed once with PBS and incubated with $0.3 \% \mathrm{H}_{2} \mathrm{O}_{2}$ in PBS for a further $20 \mathrm{~min}$ in the dark. After washing twice with PBS, $50 \mu 1$ TUNEL working solution (Beyotime Institute of Biotechnology) was added to each sample and incubated at room temperature for $1 \mathrm{~h}$. After washing twice with PBS, stop solution was added at room temperature for $10 \mathrm{~min}$. After washing twice with PBS, streptavidin-HRP working solution was added to the cells at room temperature for $30 \mathrm{~min}$. After washing twice with PBS, 3,3'diaminobenzidine solution was dropped onto the samples and incubated at room temperature for $3 \mathrm{~min}$. After washing with PBS and mounting with neutral balsam, images were captured using a Leica optical microscope (Leica Microsystems, Inc.) and routine light microscopy (magnification, x100).

Statistical analysis. Experiments were independently performed for a minimum of three times and results were qualitatively similar. Representative experiments are shown. Numerical data are presented as the mean \pm SD. Statistical analyses were performed using one-way ANOVA and the Tukey-Kramer multiple comparisons test using GraphPad Prism 6 (GraphPad Software, Inc.). $\mathrm{P}<0.05$ was considered to indicate a statistically significant difference.

\section{Results}

Expression and activity of ST3Gal3 in ovarian cancer cell lines. To identify the relationship between ST3GAL3 and MAL II in ovarian cancer cell lines, the mRNA expression levels of ST3GAL3 were investigated. The results showed that the levels of ST3Gal3 mRNA expression in HO8910PM cells was more than double that in SKOV3 cells (Fig. 1A). The protein expression levels of ST3Gal3 were also markedly higher in HO8910PM cells than in SKOV3 cells (Fig. 1B). MAL II binds to sialic acid in an $\alpha-2,3$ linkage rather than an $\alpha-2,6$ linkage, which is specific for the activity of ST3Gal3 (33). Biotinylated MAL II was used to investigate the levels of cell surface MAL II in ovarian cancer cell lines. In line with the expression levels of ST3Gal3, the $\alpha-2,3$ sialic acid linked terminal glycosylated modification was 2 -fold higher in HO8910PM cells than in SKOV3 cells (Fig. 1C and D). Collectively, the expression and activity of ST3Gal3 were significantly higher in HO8910PM cells than in SKOV3 cells.

ST3Gal3 affects paclitaxel-resistance in ovarian cancer cells. The aberrant expression and activity of ST3Gal3 in ovarian cancer cells prompted an investigation into the relationship between the expression of ST3Gal3 and paclitaxel-resistance. Paclitaxel treatments ranging from 0 to $320 \mathrm{ng} / \mathrm{ml}$ were used to induce cytotoxicity in SKOV3 and HO8910PM cells. The $\mathrm{IC}_{50}$ of paclitaxel in SKOV3 cells and HO8910PM cells were calculated to be 57.90 and $130.61 \mathrm{ng} / \mathrm{ml}$, respectively, indicating that the paclitaxel-resistance in HO8910PM cells was $>2$-fold higher than in SKOV3 cells (Fig. 2A). TUNEL assays were then used to examine paclitaxel-induced apoptosis in SKOV3 and HO8910PM cells. The results revealed that the apoptotic ratio was $\sim 2$-fold higher in SKOV3 cells than in HO8910PM cells following $48 \mathrm{~h}$ exposure to $50 \mathrm{ng} / \mathrm{ml}$ paclitaxel (Fig. 2B and C), indicating that paclitaxel-resistance was higher in HO8910PM cells than in SKOV3 cells.

ST3Gal3 knockdown sensitizes ovarian cancer cells to paclitaxel-induced apoptosis. To further investigate the role of ST3Gal3 in paclitaxel resistance, ST3Gal3 was silenced. siRNA was used to knockdown ST3Gal3 expression in SKOV3 and HO8910PM cells, followed by treatment with $20 \mathrm{ng} / \mathrm{ml}$ paclitaxel for $48 \mathrm{~h}$. The level of apoptosis was then quantified using flow cytometry. In HO8910PM cells, the knockdown of ST3GAL3 significantly increased the level of apoptosis, (si-CTRL vs. si-ST3Gal3; Fig. 3A and B). The apoptosis ratio was $\sim 3$-fold higher in si-CTRL knockdown cells treated with paclitaxel than in si-CTRL knockdown cells without paclitaxel treatment. The apoptosis ratio was $\sim 2$-fold higher in the si-ST3Gal3 cells compared with the si-CTRL cells after treated with paclitaxel (Fig. 3A and B). In SKOV3 cells, the knockdown of ST3Gal3 also increased the level of apoptosis, but not significantly 
A

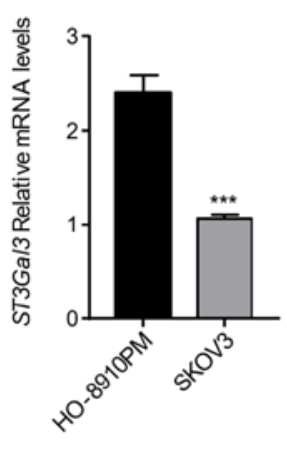

C

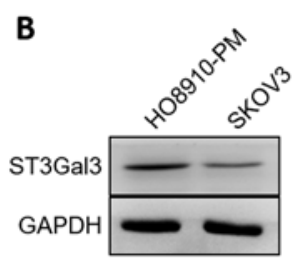

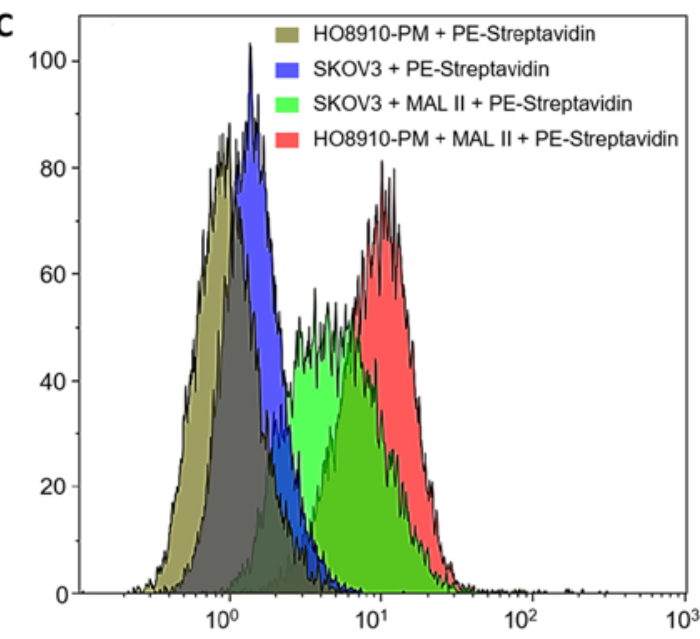

D

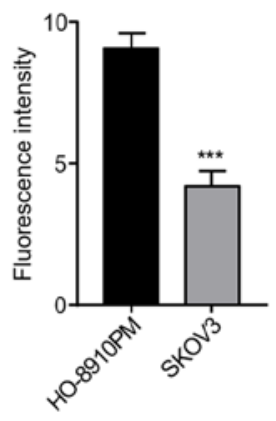

Figure 1. Expression and activity of ST3Gal3 in ovarian cancer cell lines. (A) Reverse transcription-quantitative PCR for ST3Gal3 mRNA expression levels in the ovarian cancer cell lines HO8910PM and SKOV3. (B) Western blot analysis was performed for the protein expression of ST3Gal3 in ovarian cancer cell lines. GAPDH served as the loading control. (C) Flow cytometry analysis for cell surface MAL II in the ovarian cancer cell lines. (D) Quantification of fluorescence intensity of MAL II from flow cytometry analysis. ${ }^{* * *} \mathrm{P}<0.001$ vs. HO8910PM. ST3Gal3, $\beta$-galactoside- $\alpha 2,3$-sialyltransferase III; MAL II, Maackia amurensis lectin II; PE, phycoerythrin.

A
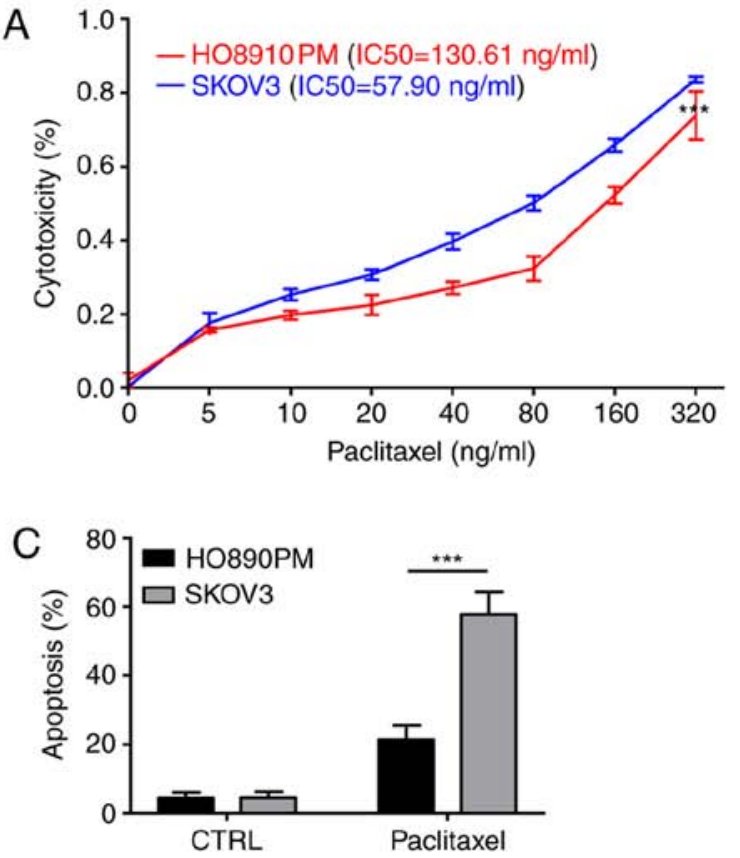

B
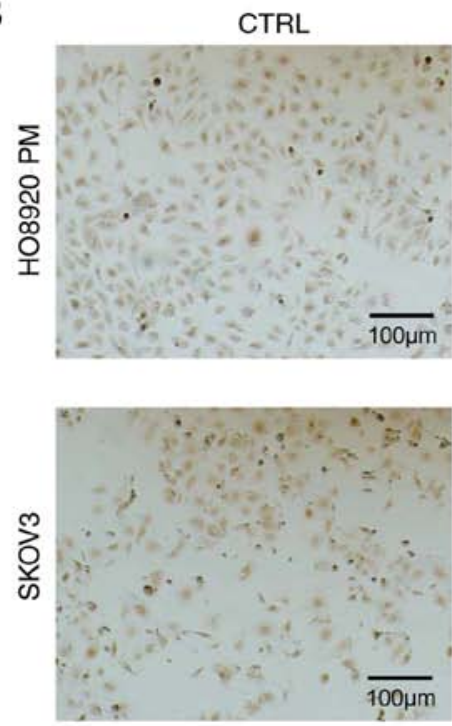

Paclitaxel
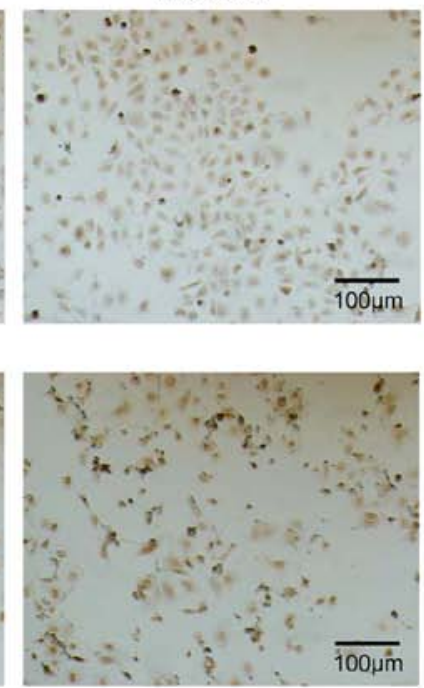

Figure 2. $\beta$-galactoside- $\alpha 2,3$-sialyltransferase III expression affects paclitaxel-resistance in ovarian cancer cells. (A) Cytotoxicity in ovarian cancer cell lines treated with the indicated concentrations of paclitaxel for $48 \mathrm{~h}$. (B) TUNEL assay was performed following $48 \mathrm{~h}$ treatment with $50 \mathrm{ng} / \mathrm{ml}$ paclitaxel in order to determine the level of apoptosis in the ovarian cancer cell lines. Representative images are shown. (C) Quantification of apoptosis rates from TUNEL assays. *** $\mathrm{P}<0.001$. CTRL, control.

(si-CTRL vs. si-ST3Gal3). The apoptosis ratio was $\sim 1.5$-fold higher in the si-ST3Gal3 cells plus paclitaxel compared with the si-CTRL cells plus paclitaxel (Fig. 3C and D). In the absence of STG3Gal3, HO8910PM cells are no longer significantly more resistant to paclitaxel than SKOV3 cells. (Fig. 3E). ST3Gal3 knockdown was found to increase paclitaxel-chemosensitivity in both SKOV3 and HO8910PM cells.

ST3Gal3 knockdown increases paclitaxel-mediated activation of caspase-8/3 signaling. The mechanism of
ST3Gal3-induced chemoresistance was investigated next. RT-qPCR and western blot results first confirmed that the si-ST3Gal3 siRNA significantly downregulated STG3Gal3 mRNA and protein levels in both HO8910PM and SKOV3 cells (Fig. 4). Paclitaxel treatment induced ST3GAL3 expression at the mRNA and protein level in HO8910PM cells (Fig. 4A and B). After the siRNA-induced downregulation of ST3Gal3, paclitaxel treatment further decreased the levels of ST3Gal3 mRNA in HO8910PM cells, rather than rescuing STG3Gal3 expression (Fig. 4A). The change in the 
A
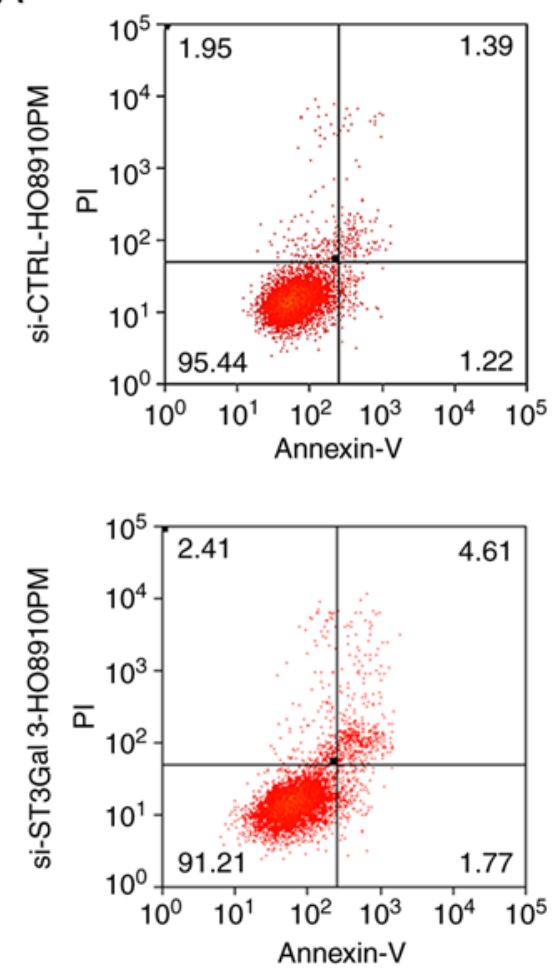

C
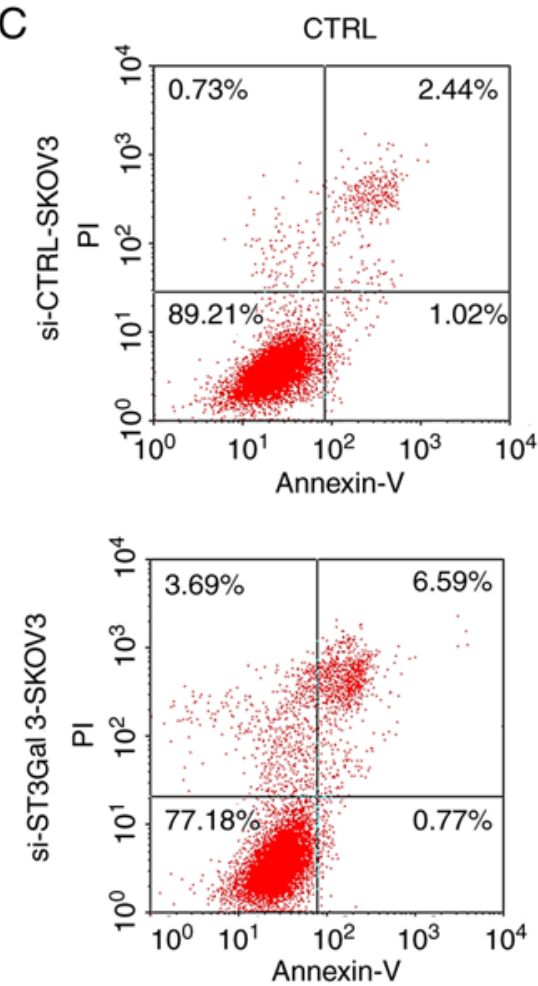
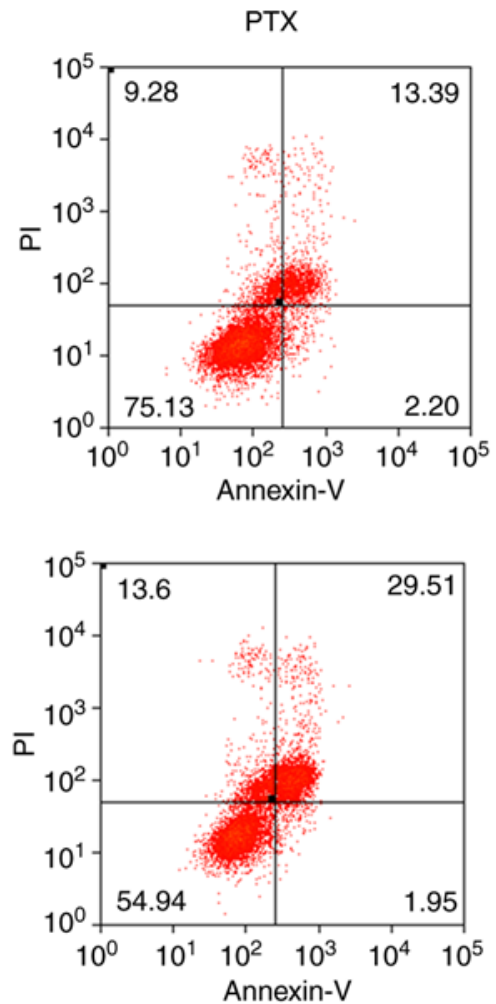

B
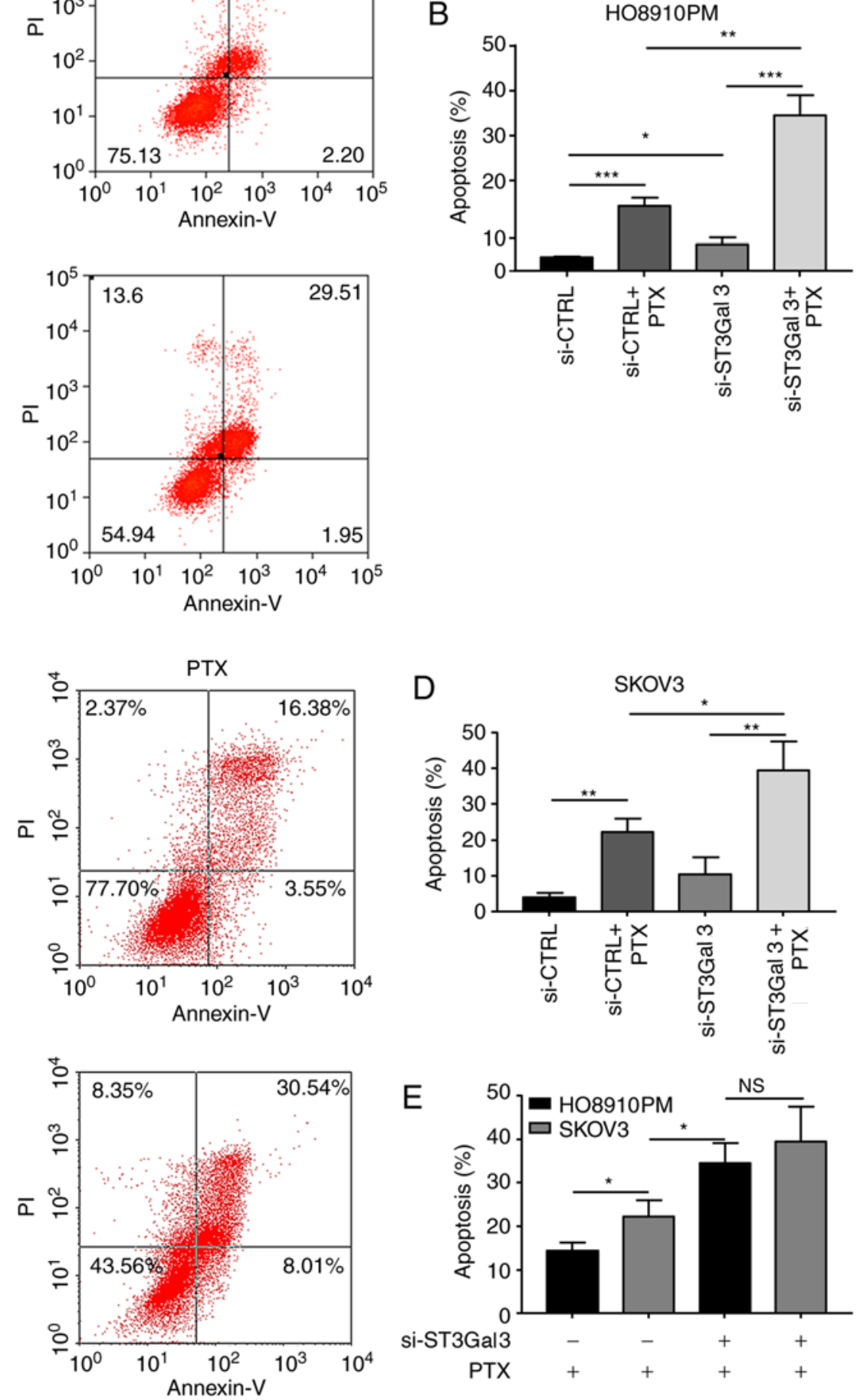

Figure 3. ST3Gal3 knockdown sensitizes ovarian cancer cells to PTX-induced apoptosis. (A) After transfection of si-ST3Gal3 into HO8910PM cells and treatment with or without $20 \mathrm{ng} / \mathrm{ml}$ PTX for $48 \mathrm{~h}$, flow cytometry was performed to determine the rates of apoptosis. (B) Quantification of apoptosis rates in HO8910PM cells. (C) After transfection of si-ST3GAL3 into SKOV3 cells and treatment with or without $20 \mathrm{ng} / \mathrm{ml}$ PTX for next $48 \mathrm{~h}$, flow cytometry was performed to determine the rates of apoptosis. (D) Quantification of apoptosis rates in SKOV3 cells. (E) Comparison of the apoptosis in HO8910PM and SKOV3 cells treated with PTX. ${ }^{*} \mathrm{P}<0.05,{ }^{* *} \mathrm{P}<0.01,{ }^{* * *} \mathrm{P}<0.001$. ST3Gal3, $\beta$-galactoside- $\alpha 2,3$-sialyltransferase III; si, small interfering; PTX, paclitaxel; CTRL, control; PI, propidium iodide.

protein level was similar (Fig. 4B). In addition, it was found that paclitaxel increased caspase- 8 and caspase- 3 protein expression levels in si-CTRL HO8910PM cells. Knockdown of ST3Gal3 alone only induced caspase-3 protein levels in HO8910PM cells compared with si-CTRL, while caspase- 8 levels were not altered (Fig. 4B). Furthermore, si-ST3Gal3 
A

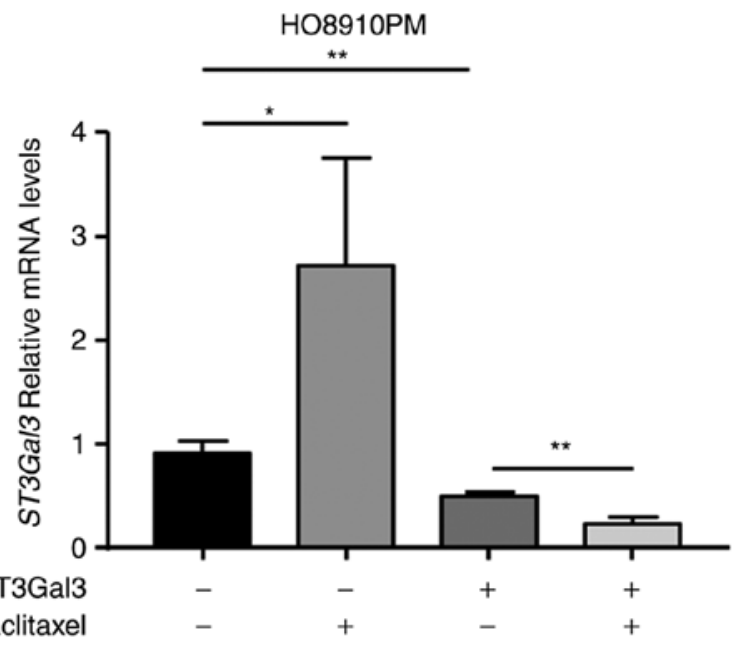

C

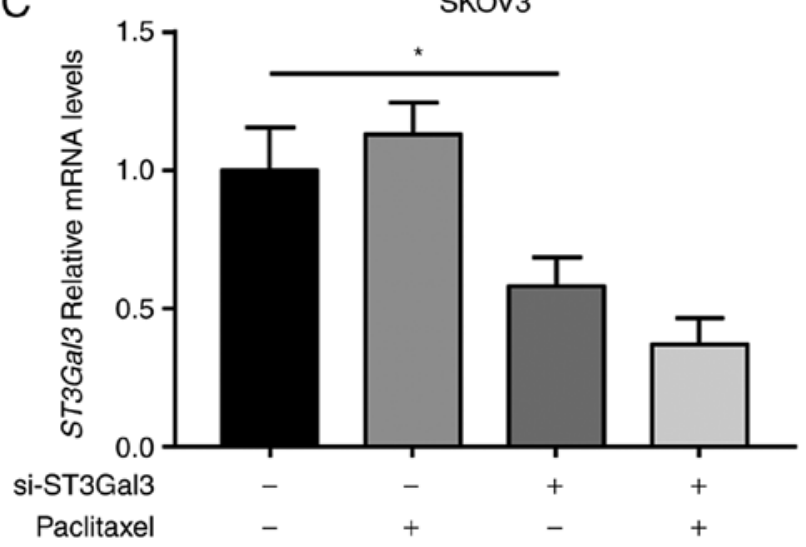

B

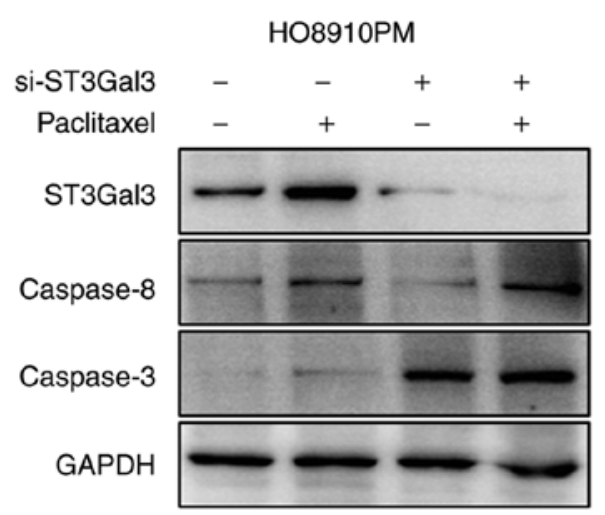

D

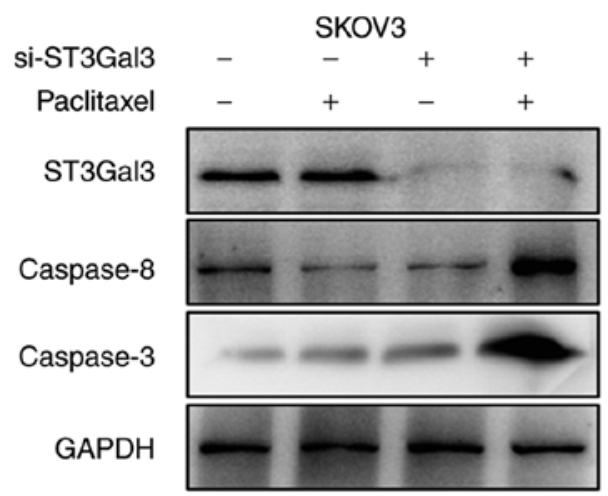

Figure 4. ST3Gal3 knockdown synergistically facilitates the paclitaxel-mediated activation of caspase-8/3. (A) Following the indicated treatments, RT-qPCR was used to determine the mRNA levels of ST3Gal3 in HO8910PM cells. (B) After the indicated treatments, western blot analysis was performed to determine the protein expression levels of ST3Ga13, caspase-3 and caspase-8 in HO8910-PM cells. GAPDH served as the loading control. (C) Following the indicated treatments, RT-qPCR was used to determine the mRNA levels of ST3Gal3 in SKOV3 cells. (D) After the indicated treatments, western blot analysis was performed to determine the protein expression levels of ST3Gal3, caspase-3 and caspase-8 in SKOV3 cells. GAPDH served as the loading control. "P<0.05 and ${ }^{* *} \mathrm{P}<0.01$, with comparisons indicated by lines. ST3Gal3, $\beta$-galactoside- $\alpha 2,3$-sialyltransferase III; RT-qPCR, reverse transcription-quantitative PCR; si, small interfering.

knockdown plus paclitaxel treatment markedly elevated caspase-8 and caspase-3 protein levels in HO8910PM cells compared with si-ST3Gal3 knockdown alone (Fig. 4B).

By contrast, paclitaxel treatment did not alter the mRNA and protein expression levels of ST3Gal3 in si-CTRL SKOV3 cells or si-ST3Gal3 SKOV3 cells (Fig. 4C and D). It was also found that paclitaxel induced caspase- 3 protein expression, but not caspase-8 expression, in si-CTRL SKOV3 cells. ST3Gal3 knockdown alone induced caspase- 3 expression, but seemed to decrease caspase-8 expression, in SKOV3 cells, compared with the si-CTRL. Finally, ST3Gal3 knockdown plus paclitaxel treatment markedly increased both caspase- 8 and caspase-3 protein level in SKOV3 cells, compared with si-ST3Gal3 knockdown alone (Fig. 4D). These results indicated that ST3Gal3 knockdown synergistically facilitated paclitaxel-mediated activation of caspase-8/3 signaling.

\section{Discussion}

Paclitaxel, a taxane-based drug, has been approved for use in a number of solid tumors for decades. However, serious side effects and chemoresistance are major obstacles that limit its clinical application, even though this drug has a remarkable response rate and increases the survival rate $(34,35)$. Therefore, focusing on reversing chemoresistance to paclitaxel remains an important and urgent focus in cancer treatment. The present results demonstrated that the expression levels of ST3Gal3 were associated with paclitaxel-resistance in ovarian cancer cell lines. Of note, paclitaxel treatment increased the mRNA and protein levels of ST3Gal3, while ST3Gal3 knockdown reduced the level of paclitaxel-induced apoptosis in ovarian cancer cells. Thus, the results of the present study suggested that ST3Gal3 may be a potential target for improving paclitaxel-based chemotherapy.

The advance in glycoinformatics and glycoproteomics has provided the needed tools to probe and understand glycosylation in development and cancer biology $(8,31)$. A number of sialyltransferases and sialylated glycoproteins have been identified as diagnostic or prognostic markers for ovarian cancer $(8,36)$. The ascites volume has been shown to be correlated with the degree of sialylation in epithelial ovarian cancer, providing a new insight into tumor progression and 
recurrence (8). Our previous study showed that the expression of sialyltransferase mRNA differs significantly among different ovarian cancer cell lines (31). In the present study, it was found that the expression of ST3Gal3 was higher in HO8910PM cells than in SKOV3 cells, indicating that more $(\alpha-2,3)$-linked sialylation events may be catalyzed on the cell surface of HO8910PM cells. Aberrant sialylations have been associated with a number of processes, including adhesion, tumor progression and metastasis (7,37,38). In addition, ST3Gal3 and ST6Gal1 play roles in cisplatin-resistance, as previously reported $(30,31,39)$. However, the combination of cisplatin and paclitaxel serves as a standard chemotherapy regimen for patients with advanced ovarian cancer $(40,41)$. Therefore, in the present study the relationship between ST3Gal3 and paclitaxel-resistance in ovarian cancer cell lines was investigated. The results of the present study showed that a higher expression level of ST3Gal3 conferred paclitaxel resistance to HO8910PM cells. Following siRNA depletion of ST3Gal3 in HO8910PM cells, a higher level of apoptosis was induced by paclitaxel compared with si-CTRL cells. There was no significant difference in paclitaxel-induced apoptosis between si-ST3Gal3 SKOV3 cells and si-ST3Gal3 HO8910PM cells. These results suggested that ST3Gal3 plays an important role in chemoresistance to paclitaxel in ovarian cancer cells.

The mechanism by which ST3Gal3 regulates paclitaxel-resistance was also explored. At a mechanistic level, paclitaxel stabilizes microtubule polymers to inhibit mitotic spindle assembly, blocking cell division and triggering apoptosis $(34,35)$. The mechanism for paclitaxel-resistance has been reported to be associated with microtubule dynamics, tubulin isotype expression and the mutation, or modifications, of tubulin-/microtubule-regulatory proteins (34). The results of the present study suggested an alternative mechanism for paclitaxel-resistance based on the following findings: i) Paclitaxel treatment upregulated ST3Gal3 expression at both the mRNA and protein level in HO8910PM cells, but not in SKOV3 cells; ii) either paclitaxel treatment alone or ST3Gal3 knockdown induced caspase-3 expression in ovarian cancer cell lines; iii) paclitaxel alone induced caspase- 8 expression in HO8910PM cells, but not in SKOV3 cells; iv) ST3Gal3 knockdown did not affect caspase-8 expression in HO8910PM cells, and even reduced caspase- 8 expression in SKOV3 cells; and v) the combination of paclitaxel treatment and ST3Gal3 knockdown markedly increased caspase- 8 and caspase- 3 expression in ovarian cancer cell lines. These results indicated that ST3Gal3 knockdown activated caspase-3, rather than caspase-8, signaling, however, paclitaxel-induced caspase signaling depended on the cell type involved. Therefore, ST3Gal3 knockdown may directly and synergistically facilitate the paclitaxel-mediated activation of caspase-3 signaling, while the activation of caspase- 8 may be dependent on the activation of caspase-3.

In conclusion, the results of the present study suggested an alternative mechanism for paclitaxel-associated chemoresistance in ovarian cancer cells. It was also indicated that aberrant ST3Gal3 expression may serve as a diagnostic and prognostic marker, and a potential chemotherapeutic target, for ovarian cancer. Future studies should be directed towards the development of sialyltransferase inhibitors; for example, high-affinity lectin may be used to block sialylated sites, or gene modification may be employed to downregulate the expression and function of sialyltransferases, with the aim to ameliorate resistance to paclitaxel-based chemotherapies.

\section{Acknowledgements}

Not applicable.

\section{Funding}

The present study was supported by the National Science Foundation of China (grant nos. 81071751 and 30770487), the Opening Project of Zhejiang Provincial Top Key Discipline of Pharmaceutical Sciences (grant no. 2016009) and the Guangzhou Science and Technology Project (grant no. 201704030059).

\section{Availability of data and materials}

The datasets used and/or analyzed during the current study are available from the corresponding author on reasonable request.

\section{Authors' contributions}

$\mathrm{XW}$ and SL conceived and designed the study, and revised the manuscripts. XZ, XY, MC, SZ and JL contributed to in vitro studies. XZ analyzed the data. XZ drafted and submitted the manuscript. All authors have read the final manuscript and approved the submission.

\section{Ethics approval and consent to participate}

Not applicable.

\section{Patient consent for publication}

Not applicable.

\section{Competing interests}

The authors declare that they have no competing interests.

\section{References}

1. Prat J; FIGO Committee on Gynecologic Oncology: Abridged republication of FIGO's staging classification for cancer of the ovary, fallopian tube, and peritoneum. Cancer 121: 3452-3454, 2015.

2. Siegel RL, Miller KD and Jemal A: Cancer statistics, 2016. CA Cancer J Clin 66: 7-30, 2016.

3. Rossing MA, Wicklund KG, Cushing-Haugen KL and Weiss NS: Predictive value of symptoms for early detection of ovarian cancer. J Natl Cancer Inst 102: 222-229, 2010.

4. Zheng X, Chen S, Li L, Liu X, Liu X, Dai S, Zhang P, Lu H, Lin Z, Yu Y and Li G: Evaluation of HE4 and TTR for diagnosis of ovarian cancer: Comparison with CA-125. J Gynecol Obstet Hum Reprod 47: 227-230, 2018.

5. Han C, Bellone S, Siegel ER, Altwerger G, Menderes G, Bonazzoli E, Egawa-Takata T, Pettinella F, Bianchi A, Riccio F, et al: A novel multiple biomarker panel for the early detection of high-grade serous ovarian carcinoma. Gynecol Oncol 149: 585-591, 2018.

6. Ferreira JA, Magalhães A, Gomes J, Peixoto A, Gaiteiro C, Fernandes E, Santos LL and Reis CA: Protein glycosylation in gastric and colorectal cancers: Toward cancer detection and targeted therapeutics. Cancer Lett 387: 32-45, 2017. 
7. Dall'Olio F, Malagolini N, Trinchera $M$ and Chiricolo $M$ : Sialosignaling: Sialyltransferases as engines of self-fueling loops in cancer progression. Biochim Biophys Acta 1840: 2752-2764, 2014.

8. Biskup K, Braicu EI, Sehouli J, Tauber R and Blanchard V: The ascites $\mathrm{N}$-glycome of epithelial ovarian cancer patients. J Proteomics 157: 33-39, 2017.

9. Rodrigues JG, Balmaña M, Macedo JA, Poças J, Fernandes Â, de-Freitas-Junior JCM, Pinho SS, Gomes J, Magalhães A, Gomes C, et al: Glycosylation in cancer: Selected roles in tumour progression, immune modulation and metastasis. Cell Immunol 333: 46-57, 2018.

10. Kuzmanov U, Musrap N, Kosanam H, Smith CR, Batruch I, Dimitromanolakis A and Diamandis EP: Glycoproteomic identification of potential glycoprotein biomarkers in ovarian cancer proximal fluids. Clin Chem Lab Med 51: 1467-1476, 2013.

11. Alley WR Jr, Vasseur JA, Goetz JA, Svoboda M, Mann BF Matei DE, Menning N, Hussein A, Mechref Y and Novotny MV: $\mathrm{N}$-linked glycan structures and their expressions change in the blood sera of ovarian cancer patients. J Proteome Res 11: 2282-2300, 2012.

12. Shetty V, Nickens Z, Shah P, Sinnathamby G, Semmes OJ and Philip R: Investigation of sialylation aberration in N-linked glycopeptides by lectin and tandem labeling (LTL) quantitative proteomics. Anal Chem 82: 9201-9210, 2010.

13. Shetty V, Hafner J, Shah P, Nickens Z and Philip R: Investigation of ovarian cancer associated sialylation changes in N-linked glycopeptides by quantitative proteomics. Clin Proteomics 9: 10, 2012.

14. Mikami M, Tanabe K, Matsuo K, Miyazaki Y, Miyazawa M, Hayashi M, Asai S, Ikeda M, Shida M, Hirasawa T, et al Fully-sialylated alpha-chain of complement 4-binding protein: Diagnostic utility for ovarian clear cell carcinoma. Gynecol Oncol 139: 520-528, 2015.

15. MatsuoK,TanabeK,IkedaM,ShibataT,KajiwaraH,MiyazawaM, Miyazawa M, Hayashi M, Shida M, Hirasawa T, et al: Fully sialylated alpha-chain of complement 4-binding protein (A2160): A novel prognostic marker for epithelial ovarian carcinoma. Arch Gynecol Obstet 297: 749-756, 2018.

16. Wen KC, Sung PL, Hsieh SL, Chou YT, Lee OK, Wu CW and Wang PH: $\alpha 2,3$-sialyltransferase type I regulates migration and peritoneal dissemination of ovarian cancer cells. Oncotarget 8 : 29013-2902, 2017.

17. Christie DR, Shaikh FM, Lucas JA IV, Lucas JA III and Bellis SL: ST6Gal-I expression in ovarian cancer cells promotes an invasive phenotype by altering integrin glycosylation and function. J Ovarian Res 1: 3, 2008.

18. Mikuła-Pietrasik J, Uruski P, Szubert S, Szpurek D, Sajdak S, Tykarski A and Książek K: Malignant ascites determine the transmesothelial invasion of ovarian cancer cells. Int J Biochem Cell Biol 92: 6-13, 2017.

19. Szender JB, Emmons T, Belliotti S, Dickson D, Khan A, Morrell K, Khan AN, Singel KL, Mayor PC, Moysich KB, et al: Impact of ascites volume on clinical outcomes in ovarian cancer: A cohort study. Gynecol Oncol 146: 491-497, 2017.

20. Orr B and Edwards RP: Diagnosis and treatment of ovarian cancer. Hematol Oncol Clin North Am 32: 943-964, 2018.

21. Zeng J, Huang H, Shan Y, Li Y, Jin Y and Pan L: The effect of CA125 nadir level on survival of advanced-stage epithelial ovarian carcinoma after interval debulking surgery. J Cancer 8 : 3410-3415, 2017

22. Jayson GC, Kohn EC, Kitchener HC and Ledermann JA: Ovarian cancer. Lancet 384: 1376-1388, 2014.

23. Webber K and Friedlander M: Chemotherapy for epithelial ovarian, fallopian tube and primary peritoneal cancer. Best Pract Res Clin Obstet Gynaecol 41: 126-138, 2017

24. Liu N, Mei L, Fan X, Tang C, Ji X, Hu X, Shi W, Qian Y, Hussain M, Wu J, et al: Phosphodiesterase 5/protein kinase G signal governs stemness of prostate cancer stem cells through Hippo pathway. Cancer Lett 378: 38-50, 2016.
25. Maji S, Panda S, Samal SK, Shriwas O, Rath R, Pellecchia M, Emdad L, Das SK, Fisher PB and Dash R: Chapter three-Bcl-2 antiapoptotic family proteins and chemoresistance in cancer. Adv Cancer Res 137: 37-75, 2018.

26. Ween MP, Armstrong MA, Oehler MK and Ricciardelli C: The role of $\mathrm{ABC}$ transporters in ovarian cancer progression and chemoresistance. Crit Rev Oncol Hematol 96: 220-256, 2015.

27. Kuchenbaecker KB, Hopper JL, Barnes DR, Phillips KA, Mooij TM, Roos-Blom MJ, Jervis S, van Leeuwen FE, Milne RL, Andrieu N, et al: Risks of breast, ovarian, and contralateral breast cancer for BRCA1 and BRCA2 mutation carriers. JAMA 317: 2402-2416, 2017.

28. Chudecka-Głaz A, Cymbaluk-Płoska A, Wężowska $M$ and Menkiszak J: Could HE4 level measurements during first-line chemotherapy predict response to treatment among ovarian cancer patients? PLoS One 13: e0194270, 2018.

29. Britain CM, Holdbrooks AT, Anderson JC, Willey CD and Bellis SL: Sialylation of EGFR by the ST6Gal-I sialyltransferase promotes EGFR activation and resistance to gefitinib-mediated cell death. J Ovarian Res 11: 12, 2018

30. Schultz MJ, Swindall AF, Wright JW, Sztul ES, Landen CN and Bellis SL: ST6Gal-I sialyltransferase confers cisplatin resistance in ovarian tumor cells. J Ovarian Res 6: 25, 2013.

31. Wang X, Zhang Y, Lin H, Liu Y, Tan Y, Lin J, Gao F and Lin S: Alpha2,3-sialyltransferase III knockdown sensitized ovarian cancer cells to cisplatin-induced apoptosis. Biochem Biophys Res Commun 482: 758-763, 2017.

32. Livak KJ and Schmittgen TD: Analysis of relative gene expression data using real-time quantitative PCR and the 2(-Delta Delta $\mathrm{C}(\mathrm{T})$ ) method. Methods 25: 402-408, 2001

33. Geisler C and Jarvis DL: Effective glycoanalysis with Maackia amurensis lectins requires a clear understanding of their binding specificities. Glycobiology 21: 988-993, 2011.

34. Jordan MA and Wilson L: Microtubules as a target for anticancer drugs. Nat Rev Cancer 4: 253-265, 2004.

35. Orr GA, Verdier-Pinard P, McDaid H and Horwitz SB: Mechanisms of Taxol resistance related to microtubules. Oncogene 22: 7280-7295, 2003

36. Bennun SV, Hizal DB, Heffner K, Can O, Zhang $\mathrm{H}$ and Betenbaugh MJ: Systems glycobiology: Integrating glycogenomics, glycoproteomics, glycomics, and other 'omics data sets to characterize cellular glycosylation processes. J Mol Biol 428: 3337-3352, 2016

37. Wu J, Xie X, Nie S, Buckanovich RJ and Lubman DM: Altered expression of sialylated glycoproteins in ovarian cancer sera using lectin-based ELISA assay and quantitative glycoproteomics analysis. J Proteome Res 12: 3342-3352, 2013.

38. Cheng WK and Oon CE: How glycosylation aids tumor angiogenesis: An updated review. Biomed Pharmacother 103: 1246-1252, 2018.

39. Zhang X, Pan C, Zhou L, Cai Z, Zhao S and Yu D: Knockdown of ST6Gal-I increases cisplatin sensitivity in cervical cancer cells. BMC Cancer 16: 949, 2016.

40. Goldberg JM, Piver MS, Hempling RE and Recio FO: Paclitaxel and cisplatin combination chemotherapy in recurrent epithelial ovarian cancer. Gynecol Oncol 63: 312-317, 1996.

41. du Bois A, Lück HJ, Meier W, Möbus V, Costa S, Richter B, Warm M, Bauknecht T, Schröder W, Olbricht S, et al: Carboplatin/paclitaxel versus cisplatin/paclitaxel as first-line chemotherapy in advanced ovarian cancer: An interim analysis of a randomized phase III trial of the Arbeitsgemeinschaft Gynäkologische Onkologie Ovarian Cancer Study Group. Semin Oncol 24 (5 Suppl 15): S15-44-S15-52, 1997.

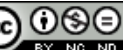

This work is licensed under a Creative Commons Attribution-NonCommercial-NoDerivatives 4.0 International (CC BY-NC-ND 4.0) License. 